\title{
Les Grands Barrages et la crise environnementale en Afrique
}

\author{
Jaime LLambias-Wolff and Shally B. Gachuruzi
}

\begin{abstract}
This article seeks to demonstrate that although large dams are incontestably considered "engines" of development, their success is not always assured. They are nearly always accompanied by negative and harmful effects on the environment, which in turn mortgages the profitability of the sought-after development.
\end{abstract}

\section{Introduction}

Au cours de ces quatre dernières décennies, les principes de développement ont reposé sur la modernisation, l'urbanisation et une rapide industrialisation des pays du Tiers Monde. La mobilisation des ressources tant nationales qu'internationales avait pour objet de doter ces pays d'une infrastructure capable de moderniser l'appareil économique de l'État et de renforcer leur potentiel productif. Dans cette logique, plusieurs pays du Tiers Monde ont mis en place des programmes de modernisation agro-industrielle pour combler le retard accusé par rapport aux pays avancés et assurer les conditions d'un développement rapide et soutenu de leur économie. C'est dans cette perspective de rattrapage que de nombreux pays $d$ 'Afrique ont cherché au lendemain de leur indépendance, à construire une autonomie énergétique nécessaire pour le développement des activités productives et modernes. Avec le soutien des agences et capitaux étrangers, ils ont ainsi misé sur des méga-projets. La multiplicité d'importants barrages sur les fleuves africains en est une illustration. Citons à titre d'exemple le barrage d'Inga construit sur le fleuve

Professor Jaime LLambias-Wolff teaches sociology at York University

Dr. Shally Gachuruzi is a research associate at CRS, York University, with interests in forced migration, the environment and development.
Zaire au Zaïre, celui de Kariba construit en 1958 sur le fleuve Zambèze à la frontière entre la Zambie et le Zimbabwe, le barrage d'Akossomboérigé sur la Volta au Ghana en 1964, le barrage de Kainji construit en 1968 sur le Niger au Nigeria, le haut barrage d'Assouan construit en 1969 sur le Nil en Égypte dont la retenue d'eau s'étend jusque dans les terres soudanaises et enfin celui de Kossou construit en 1969 sur le Bandama en Côte d'Ivoire et bien d'autres. Le tableau ci-après présente quelques aménagements hydroélectriques africains.

\begin{tabular}{|cll|}
\hline \multicolumn{3}{c}{ Tableau 1 } \\
Quelques Aménagements \\
Hydroélectriques Africains \\
& & \\
Date & Pays & Fleuve \\
1970 & Côte d'Ivoire & Bandama \\
1969 & Egypte & Nil \\
1968 & Nigeria & Niger \\
1964 & Ghana & Volta \\
1958 & Zambie & Zambèze \\
Source: Lassailly 1983, 48. & \\
\hline
\end{tabular}

Malheureusement, la contribution du secteur moderne à la croissance économique n'a guère répondu aux attentes qu'avait suscitées l'industrialisation accélérée. Cette croissance est restée faible si non stagnante durant plusieurs années et ses effets tant directs qu'indirects sur les revenus n'ont correspondu dans aucun cas aux prévisions.

Cet article vise à démontrer que malgré que les mégaprojets soient incontestablement considérés comme des moteurs de développement économique, leur succès n'est pas toujours évident. Ils s'accompagnent presque toujours des effets pervers qui au lieu de favoriser la population locale, posent un ensemble de problèmes graves d'ordre socio-économique et environnementale.

\section{Impacts négatifs d'ordre environnemental}

Les aménagements de grands barrages perturbent gravement l'écosystème de vastes régions. Ils s'accompagnent souvent en amont de la formation de vastes lacs artificiels, véritables mers intérieures qui inondent parfois des régions entières. Citons à titre d'exemple le lac Volta dont la superficie de 9 $000 \mathrm{~km}^{2}$ occupe $3,5 \%$ du territoire ghanéen ou encore le lac Nasser en Égypte qui couvre une superficie de $6500 \mathrm{~km}^{2}$ et le lac Kariba au Zimbabwe qui couvre $5300 \mathrm{~km}^{2}$. Le lac Kossou quant à lui, couvre une superficie de $1750 \mathrm{~km}^{2}$ (Lassailly 1983, 48), soit approximativement la taille du lac St-Jean au Québec. La situation est beaucoup plus alarmante en Inde et en Chine où seuls deux barrages (Narmada et Three Gorges) ont inondé une superficie de 135 348 ha. Généralement dans pareil cas, un écosystème terrestre devient brusquement aquatique avec toutes les transformations que cela suppose.

Parmi les conséquences néfastes d'ordre écologique, la salinisation occupe aussi une place importante. La citation suivante rappelle les effets écologiques dûs au phénomène de la salinisation:

L'irrigation implantée dans les régions semi-arides dégrade facilement et rapidement les terres cultivables si l'on ne prend pas de précautions pour écouler les sels sodiques généralement répartis sous forme de grandes tâches sur les sols de ces régions et dans les eaux rete nues par les barrages. (Schwarz 1989, 194-95)

Cette façon de voir les choses corrobore ce que Barré et Godet appellent "effet d'Assouan" par référence au barrage d'Assouan en Egypte où

il apparaît aujourd'hui de multiples problèmes d'une dimension telle que les inconvénients présents et futurs 
(salinisation des terres, moindre fertilisation en aval par le limon des crues, la chute drastique de la pêche dans le delta du Nil) pourraient l'emporter sur les avantages passés du fait de l'irrigation. En effet, la mauvaise exploitation et la surexploitation des terres jointes à l'érosion, la salinisation et la latérisation risquent d'entraîner finalement l'extension de la désertification. (Barré et Godet 1983, 508)

Radheshyam $(1990,27)$ partage ce point de vue quand il affirme que l'augmentation de la salinité des surfaces des terres cultivables à cause de l'irrigation rend le sol improductif. La nature et la quantité d'argile provenant du drainage souterrain rendent le sol vulnérable au sodium.

Dans le même ordre d'idée, Philippe et Taoufik $(1986,23)$ font remarquer que "les terres irriguées ne sont pas un oasis de verdure, mais une zone désertique". En effet, quand les labours sont très profonds, les terres se dessèchent très vite et facilitent les remontées salines. La mince couche de terre recouvrant le sol fertile s'appauvrit exigeant ainsi une régénération des matières organiques dont l'épuisement menace l'agriculture.

En rapport avec les effets de la salinité du sol, Larson $(1993,86)$ rapporte l'exemple de Shanghai où entre 19781979,1333 ha de récolte du riz ont été détruits à cause du taux très élevé de la salinité due à l'irrigation, soit une perte totale de $\$ 4963000$.

Du reste, ces grands barrages risquent de mettre fin à des habitudes traditionnelles de conservation du milieu. En effet, l'exploitation familiale constitue la meilleure prévention écologique. Comme l'indique Dupriez $(1982,108)$ qui, en se servant de l'expérience de la culture associée pratiquée chez les Bashis dans la région montagneuse du Kivu au Zaïre et des Sereres

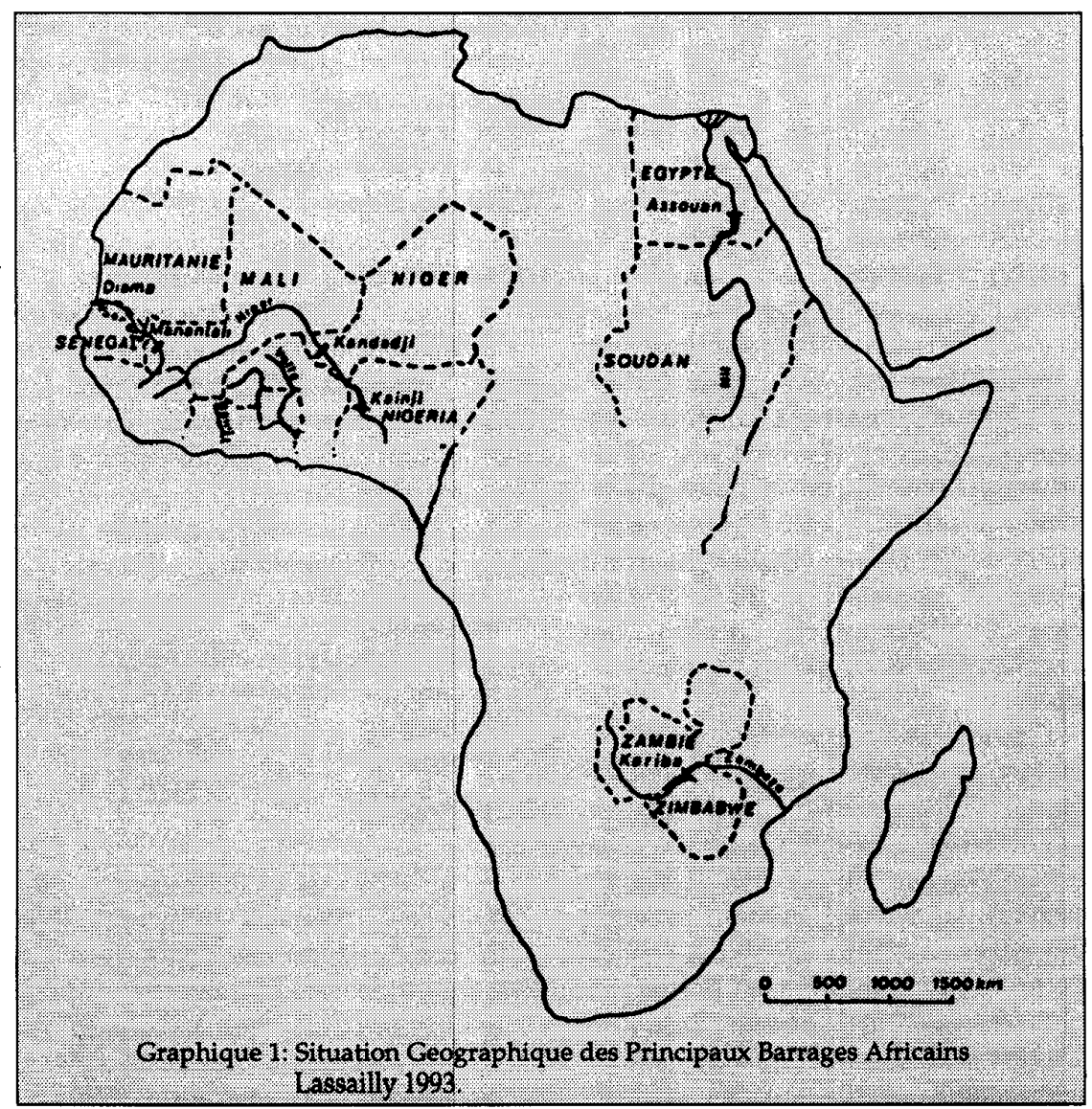

au Sénégal, ces peuples réussissent à protéger leur espace écologique. Ils savent par exemple que l'arbre est d'une importance capitale dans la conservation du sol, parce qu'il atténue les effets du climat en créant sous son couvert ou à proximité un microclimat tout en protégeant le sol contre l'érosion.

Par exemple, les collectivités forestières de l'Asie et de bien d'autres communautés locales des pays du Tiers Monde entretiennent des liens complexes avec la forêt. La diversité et la richesse caractérisant la connaissance qu'ont ces collectivités indigènes des ressources naturelles trouvent leur expression dans les technologies et valeurs traditionnelles acquises à travers des siècles. Selon le rapport de la commission sur l'environnement et le développement durable,

on a acquis ce savoir par tâtonnements au gré de l'interaction avec la nature au fil des siècles, connaissances qui se sont accumulées et qui se sont transmises de générations en génération. Ce qui est surtout en cause, c'est une exploitation diversifiée et durable des ressources naturelles. Une agriculture itinérante a été écologiquement durable sur un grand nombre de générations. Ainsi, l'environnement a façonné les populations. Leur économie, leur mode de vie, bref leur culture, traduisent par dessus tout une adaptation de l'humain au milieu naturel. (CRDI 1992)

Les grands barrages, responsables de la disparition du couvert végétal et forestier soit par inondation, soit par la coupe permettant l'aménagement des routes d'accès aux barrages, risquent de contribuer à l'abaissement de la nappe phréatique et de perturber négativement le milieu naturel.

La coupe du bois affecte aussi sérieusement les agriculteurs; non seulement elle augmente les inondations et les glissements de terrain rendant ainsi la terre infertile, mais aussi elle endommage les ressources forestières indispensables à la survie des habitants qui vivent aux alentours du projet. On sait par exemple que pour la population rurale, la seule source d'énergie dont elle dispose est le bois. Même dans les 
grands centres urbains, l'énergie du bois supplante de loin l'hydroélectricité. A Kinshasa/Zaïre par exemple, plus de $60 \%$ de la population sur un total de 4 millions d'habitants, utilise les ressources forestières ou le charbon de bois pour la cuisson des aliments.

La construction des grands barrages ne menace pas seulement la vie humaine, elle met aussi en péril la vie sauvage dont dépend souvent la population en détruisant leur habitat naturel. Comme l'indique Radheshyam $(1990,27)$, le changement de courant et de température de l'eau dans les turbines affecte la vie aquatique et dérange le libre passage des poissons. Ceci a pour conséquence un ralentissement de l'industrie de la pèche provoquant hélas une baisse de revenus des pêcheurs.

Un autre fait à souligner, parce que bien souvent ignoré à propos de construction des barrages est le risque qu'ils peuvent provoquer en cas d'erreur d'ingénierie. En effet, advenant une rupture, les eaux du barrage pourraient provoquer des pertes humaines importantes, destruire des habitats sauvages et bouleverser l'écosystème du milieu. A titre d'exemple, en 1963 le barrage de Vaient en Italie a causé un glissement de terrain en entraînant des inondations importantantes et la mort de 4 milles personnes.

La plus grande catastrophe dont il faut redouter les conséquences est celle des tremblements de terre causés par les grands barrages. Comme l'indique Williams $(1993,27)$, "1'un de plus important critère de structure d'un barrage est l'estimation d'une éventuelle accélération de tremblement de terre". Il cite l'exemple du barrage de Koyna en Inde qui, en Décembre 1967 a provoqué un séisme d'approximativement 6.0 degrés à l'échelle de Richter. Ce séisme endommagea sérieusement le barrage, tua 200 personnes, en blessa 1500 sans compter de milliers des sans-abri. Plus de $80 \%$ des maisons de Koyonanagar ont été complètement détruites ou rendues inhabitables.

Comme on peut le constater, les grands barrages ne constituent pas nécessairement un choix technologique gagnant. Il convient de souligner qu'il est impératif de se fier aux études d'impacts environnementaux des grands barrages avant leur mise en chantier.

\section{Conclusion}

En guise de conclusion, soulignons que les grands barrages occupent une place de choix dans tout processus de développement industriel. Considérés sous l'angle de leur contribution au modernisme, ces mégaprojets offrent un incontestable intérêt. Mais, bien qu'ils soient indéniablement considérés comme un moteur de développement, ils provoquent souvent des impacts négatifs notamment d'ordre environnemental.

Ainsi, au lieu de promouvoir les économies de la région et d'améliorer les conditions de vie des villageois, ils accélèrent la prolétarisation des paysans qui sont victimes de la dégradation de leurs terroirs les condamnant ainsi à un refuge incertain.

\section{Références}

CRDI. 1992. Pour l'amour de la terre. Montréal: Éditions, Méridien.

Dupriez Hugues. 1982. Paysons d'Afrique noire. Paris: Karthala.

Godet, M., et Barré R. 1983. "Le développement hypothequé", dans Revue du Tiers Monde, vol. 23.

Larson, Joseph. 1993. “Downstream, environmental impacts," in Damming the Three Gorges. Probe International.

Lassailly, Véronique. 1983. "L'espace géographique, Grands barrages africains et prise en compte des populations locales". Paris: Laboratoire de sociologie et de géographie africaines, CNRS.

Philippe E., et Taufik. 1986. Enjeux de l'après barrage. Paris: Karthala.

Radheshyam, Duria. 1990. Environmental impact of Narmada Sagar project. Ashish Publishing House.

Schwarz, Alf. 1989. L'industrie de la sécheresse. Québec, Québec: CRAD, Université Laval.

Williams, Philippe. 1993. "Dam Safety Analysis", in Damming the Three Gorges, Probe International. $\square$
New Director of Centre for Refugee Studies Announced

Anne Bayefsky, a professor in the Faculty of Law at the University of Ortawa, has been named the new director of York's Centre for Refugee Srudies for a three-year tern, effective July 1. She will succeed David Dewitt. who has been serving as the centre's interim director.

"The Centre for Refugee Studies has a world class reputation, and the opportunity to expand and carry on its work is an honour and a challenge," said Bayefsky.

The author of a number of publications in the fields of international protection of human rights, internarional law, constitutional law, civil liberties, anti-discrimination law, women's rights, and jurisprudence, Bayefkky will bring her wide knowledge and expertise to York. She will also be joining York's Department of Political Science, (Faculty of Arts).

The range of Bayefsly's involvement in research activities is impressive indeed. She has been distinguished for a number of her works on issues of hurnan rights. Currently, she holds a grant in 1995-96 from the McArthur Foundation's Program on Peace and International Cooperation for projects that make a positive difference in facing the numerous challenges to peace, security and human dignity in the contemporary world. In 1992, she received the Bora Laskin National Fellowship in Human Rights Research, Canada's premier human rights fellowship, which is administered by the Social Sciences and Humanities Research Council of Canada (SSHRC)

She is on advisory boards and committees of a number of prominent international bodles, and has participated in many Canadian delegations to United Nations conferences and commissions on human rights.

I' m very excited to come to York as director of the Centre of Refugee Snudies. It's a dynamic and vibrant tesearch centre- a unique place for thestudy and examination of refugee issues" said Bayefsky.

Among other activities, Bayefshy's upcoming projects include "exploring and cementing the connections and relationships between refugee and human rights issues?

Sokrce York Univenity Gazetts. April 17, 1996 Reprinted with permission. 
Refuge

York Lanes Press

Centre for Refugee Studies

Suite 351, York Lanes

York University

4700 Keele Street, North York

Ontario, Canada M3J 1P3

Phone: (416) 736-5843

Fax: (416) 736-5837

Internet: refuge@yorku.ca

\section{SUMmer Course ON REFUGEE ISSUES}

TORONTO • JUNE 19-27, 1996

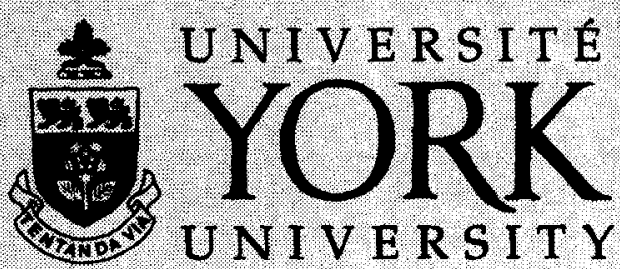

The Centre for Refugee Studies' Summer Course on Refugee Issues is intended to deepen understanding, broaden perspectives, and provide fresh insights on refugee protection and solutions to people who are already familiar with refugee issues and who have a degree or equivalent experience. In nine days, which includes one pause day, the Course offers an overview of refugee issues plus an opportunity to explore current topics with international experts.

Module 1: Comprehensive Overview (Four intensive days-Wednesday to Saturday)

- Nation States and Refugees • Social Demography and Refugee Movements

- Role of the UNHCR and International Organizations - Law and Refugee Status

- Gender Issues in Refugee Affairs * Repatriation and Development Issues

Module 2: Special Topics (Four intensive days-- Monday to Thursday)

- Practical Experience from Refugee Repatriations - The Future of Refugee Protection in Africa

- The Role and Potential of Regional Bodies in the Protection of Refugees and Internally Displaced Persons in the Americas - Racism and Public Policy - Class, Wealth and Other Factors in Settlement of Refugees - The Right to Seek Asylum and Asylum Sharing Agreements

- Whither Europe? Dealing with an Emergency

Fees for Full Course, inclusive of materials: \$700; Late registration after April 30, 1996: \$750

Subsidised to $\$ 400$ for nongovernmental participants: $\$ 450$ after April $30,1996$.

Fees for Half Course, inclusive of materials: $\$ 350$; Late registration after April 30, 1996: $\$ 400$

Subsidised to \$250 for nongovernmental participants; $\$ 325$ after April 30, 1996.

Participants are encouraged to take the full eight-day program, however, it is possible to take only Module 1. Food and accommodation are extra. Reasonably priced accommodation and food are available on campus.

For further information and registration, please contact:

Dr. Tom Clark, Summer Course Coordinator, Centre for Refugee Studies

Suite 322, York Lanes, York University, North York ON M3I 1P3 Canada

Tel: (416) 736-5663 · Fax: (416) 736-5837 - Internet: telark@yorku.ca 